\title{
On the electrostatic potential and electric field of a uniformly charged disk
}

\author{
V Bochko ${ }^{1}$, Z K Silagadze ${ }^{1,2}$ \\ ${ }^{1}$ Novosibirsk State University, Novosibirsk 630090, Russia \\ E-mail: v.bochko@g.nsu.ru \\ ${ }^{2}$ Budker Institute of Nuclear Physics, Novosibirsk 630 090, Russia \\ E-mail: Z.K.Silagadze@inp.nsk.su
}

\begin{abstract}
We calculate the electrostatic potential and electric field of a uniformly charged disk everywhere in space. This electrostatic problem was solved long ago, and its gravitational analogue - even earlier. However, it seems that physics students are not aware of the solution, because it is not presented in textbooks. The purpose of the present article is to fill this gap in the pedagogical literature.
\end{abstract}

Submitted to: Eur. J. Phys. 


\section{Introduction}

The problem of finding the electric field on the axis of symmetry of a uniformly charged circular disk is considered in many introductory textbooks on classical electrodynamics (see, for example, [1, 2, 3, 4, 5, 6]). Naturally, students may wonder how the electric field (or the electrostatic potential) can be found in a more general case for points that do not lie on the axis of symmetry. Surprisingly, students will not find the answer even in more advanced textbooks [7, 8, 9, 10, 11, 12, 13, 14, 15, 16]. In [12] a hint is given without any further details that the electric field around a homogeneously charged disk generally requires expression in terms of elliptic integrals.

The desired general answer in terms of elliptic integrals can be found in a comprehensive French treatise [17], in Russian textbooks on potential theory [18, 19], or in special scientific literature [20, 21, 22]. However, we are afraid that not one of them are especially accessible to the average student.

In an interesting exercise 2.53 in [6] (marked as a difficult four-star problem), it is proposed to show that the radial component of the electric field of a uniformly charged disk, at a point $P$ located at a distance $\eta R$ from the center of the disk (where $0 \leq \eta<1$ and $R$ is the radius of the disk) and at an infinitesimal distance away from the plane of the disk, has the following form

$$
E_{r}=\frac{\sigma}{2 \pi \epsilon_{0}} \int_{0}^{\pi / 2} \ln \left(\frac{\sqrt{1-\eta^{2} \sin ^{2} \theta}+\eta \sin \theta}{\sqrt{1-\eta^{2} \sin ^{2} \theta}-\eta \sin \theta}\right) \cos \theta d \theta,
$$

where $\sigma$ is surface charge density on the disk.

Instead of using cylindrical coordinates associated with the center of the disk, which might seem natural due to the symmetry of the problem, a hint in the book suggests to divide the disk into infinitely small wedges centered at point $\mathrm{P}$, as shown in Fig,1, then find the sum of the fields from the two opposite wedges, and finally integrate over the angle $\theta$.

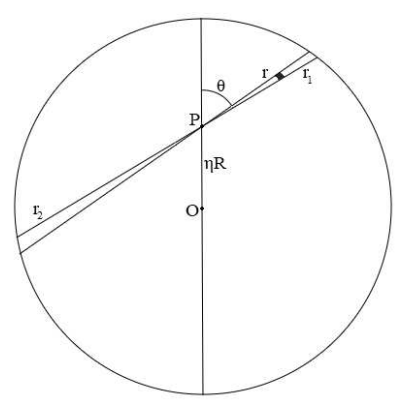

Figure 1. Dividing the disk into infinitely small wedges centered at point $\mathrm{P}$. The black patch at distance $r$ from $P$ is a wedge element containing the charge $d q=\sigma r d r d \theta$.

This clever trick can already be found in [23], and it was again discovered in the form of the so-called strip function technique in [24] when studying the electrostatics 
and magnetostatics of a conducting disk. This method (without much emphasis) was used in 20] when calculating the gravitational potential due to uniform disk.

The purpose of this note is to demonstrate that with a certain level of moderate mathematical complexity (basic knowledge of elliptic integrals), the same technique allows to find the electrostatic potential and electric field of a uniformly charged disk everywhere in space, and not just on the disk.

\section{Electrostatic potential on the disk}

An element of the right wedge in Fig,1, at distance $r$ from $P$, contains an amount of charge $d q=\sigma r d r d \theta$ and, thus, creates at $P$ the electrostatic potential $d q /\left(4 \pi \epsilon_{0} r\right)=$ $\sigma d r d \theta /\left(4 \pi \epsilon_{0}\right)$. The contribution of the entire right wedge is then

$$
d \phi_{R}(P)=\frac{\sigma d \theta}{4 \pi \epsilon_{0}} \int_{0}^{r_{1}} d r=\frac{\sigma r_{1}}{4 \pi \epsilon_{0}} d \theta .
$$

The same consideration applies to the left wedge with the result

$$
d \phi_{L}(P)=\frac{\sigma r_{2}}{4 \pi \epsilon_{0}} d \theta
$$

When $\theta$ changes from 0 to $\pi / 2$, a half of the disk's area is covered. It is evident from the symmetry that another half gives the same contribution to the potential at $P$. Therefore, finally

$$
\phi(P)=\frac{\sigma}{2 \pi \epsilon_{0}} \int_{0}^{\pi / 2}\left[r_{1}(\theta)+r_{2}(\theta)\right] d \theta .
$$

The functions $r_{1}(\theta)$ and $r_{2}(\theta)$ can be found from Fig,2. Namely, using the law of cosines,

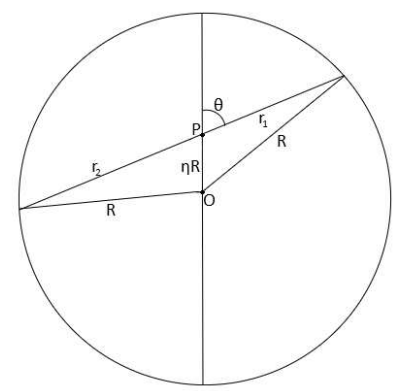

Figure 2. Illustration of the calculation of $r_{1}(\theta)$ and $r_{2}(\theta)$ from the law of cosines.

we get

$$
R^{2}=r_{1}^{2}+\eta^{2} R^{2}+2 \eta r_{1} R \cos \theta=r_{2}^{2}+\eta^{2} R^{2}-2 \eta r_{2} R \cos \theta .
$$

Solving quadratic equations (5) for $r_{1}$ and $r_{2}$, we get (note that $\eta \cos \theta<\sqrt{1-\eta^{2} \sin ^{2} \theta}$, since $\eta<1$ )

$$
r_{1}=R\left[\sqrt{1-\eta^{2} \sin ^{2} \theta}-\eta \cos \theta\right], r_{2}=R\left[\sqrt{1-\eta^{2} \sin ^{2} \theta}+\eta \cos \theta\right]
$$


Therefore, (4) takes the form

$$
\phi(P)=\frac{\sigma R}{\pi \epsilon_{0}} \int_{0}^{\pi / 2} \sqrt{1-\eta^{2} \sin ^{2} \theta} d \theta=\frac{\sigma R}{\pi \epsilon_{0}} E(\eta),
$$

where

$$
E(k)=\int_{0}^{\pi / 2} \sqrt{1-k^{2} \sin ^{2} \theta} d \theta
$$

is the complete elliptic integral of the second kind [25, 26].

For the radial component of the electric field at the point $P$ we get from (17) the following expression

$$
E_{r}(P)=-\frac{1}{R} \frac{\partial \phi_{P}}{\partial \eta}=\frac{\sigma \eta}{\pi \epsilon_{0}} \int_{0}^{\pi / 2} \frac{\sin ^{2} \theta}{\sqrt{1-\eta^{2} \sin ^{2} \theta}} d \theta=\frac{\sigma}{\pi \epsilon_{0}} \frac{K(\eta)-E(\eta)}{\eta}
$$

where

$$
K(k)=\int_{0}^{\pi / 2} \frac{d \theta}{\sqrt{1-k^{2} \sin ^{2} \theta}}
$$

is the complete elliptic integral of the first kind and at the last step we have used the relation [25, 26]

$$
\frac{d E(k)}{d k}=\frac{E(k)-K(k)}{k} \text {. }
$$

At first sight (1) and (9) look different. However, we can write (1) in the form

$$
E_{r}=\frac{\sigma}{2 \pi \epsilon_{0}}[I(\eta)-I(-\eta)]
$$

where the integral $I(\eta)$ can be transformed using integration by parts as follows:

$I(\eta)=\int_{0}^{\pi / 2} \ln \left[\sqrt{1-\eta^{2} \sin ^{2} \theta}+\eta \cos \theta\right] d \sin \theta=\ln \sqrt{1-\eta^{2}}+\int_{0}^{\pi / 2} \frac{\eta \sin ^{2} \theta}{\sqrt{1-\eta^{2} \sin ^{2} \theta}} d \theta$,

and this makes clear that (11) and (9) are, in fact, equivalent.

\section{Electrostatic potential above or below the disk}

Now let's assume that the point $P$ in Fig, 1 is just the projection on the disk of the point where we want to find the electrostatic potential. Then instead of (2) and (3) we will have

$$
d \phi_{R}=\frac{\sigma d \theta}{4 \pi \epsilon_{0}} \int_{0}^{r_{1}} \frac{r}{\sqrt{r^{2}+z^{2}}} d r=\frac{\sigma d \theta}{4 \pi \epsilon_{0}}\left(\sqrt{r_{1}^{2}+z^{2}}-|z|\right)
$$

and

$$
d \phi_{L}=\frac{\sigma d \theta}{4 \pi \epsilon_{0}} \int_{0}^{r_{2}} \frac{r}{\sqrt{r^{2}+z^{2}}} d r=\frac{\sigma d \theta}{4 \pi \epsilon_{0}}\left(\sqrt{r_{2}^{2}+z^{2}}-|z|\right),
$$


Therefore, in this case the electrostatic potential takes the form

$$
\phi(\eta R, z)=\frac{\sigma}{2 \pi \epsilon_{0}} \int_{0}^{\pi / 2}\left(\sqrt{r_{1}^{2}+z^{2}}+\sqrt{r_{2}^{2}+z^{2}}-2|z|\right) d \theta
$$

where $r_{1}(\theta)$ and $r_{2}(\theta)$ are given by (6) and it is clear from these expressions that $r_{2}(\pi-\theta)=r_{1}(\theta)$. Then

$$
\int_{0}^{\pi / 2} \sqrt{r_{2}^{2}+z^{2}} d \theta=\int_{\pi / 2}^{\pi} \sqrt{r_{1}^{2}+z^{2}} d \theta
$$

and (16) can be rewritten as

$$
\phi(\eta R, z)=\frac{\sigma}{2 \pi \epsilon_{0}}\left(\int_{0}^{\pi} \sqrt{r_{1}^{2}+z^{2}} d \theta-\pi|z|\right) .
$$

To calculate the integral in (18), it is convenient to introduce the angle $\psi$ shown in Fig 3 [23] and use $\phi=\pi / 2-\psi$ as the new integration variable instead of $\theta$.

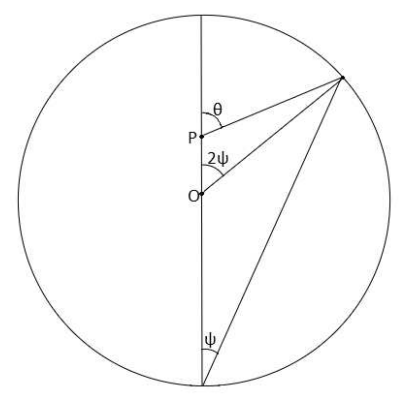

Figure 3. The meaning of the angle $\psi$.

Since $O P=\eta R$, it is clear from the figure Fig 3 that

$$
\tan \theta=\frac{R \sin 2 \psi}{R \cos 2 \psi-\eta R}=\frac{\sin 2 \psi}{\cos 2 \psi-\eta},
$$

and therefore

$$
d \theta=2 \frac{1-\eta \cos 2 \psi}{1+\eta^{2}-2 \eta \cos 2 \psi} d \psi=-2 \frac{1+\eta \cos 2 \phi}{1+\eta^{2}+2 \eta \cos 2 \phi} d \phi .
$$

Fig. 3 shows that when $\theta$ changes from 0 to $\pi, \psi$ changes from 0 to $\pi / 2$ and, correspondingly, $\phi$ changes from $\pi / 2$ to 0 . Therefore, the integral in (18) can be written in the following way:

$$
\int_{0}^{\pi} \sqrt{r_{1}^{2}+z^{2}} d \theta=\frac{2 R \sqrt{(1+\eta)^{2}+z^{2} / R^{2}}}{(1+\eta)^{2}} \int_{0}^{\pi / 2} \frac{\left(1+\eta-2 \eta \sin ^{2} \phi\right)\left(1-k^{2} \sin ^{2} \phi\right)}{\left(1-n^{2} \sin ^{2} \phi\right) \sqrt{1-k^{2} \sin ^{2} \phi}} d \phi .
$$

Here we have used $r_{1}^{2}=R^{2}\left(1+\eta^{2}-2 \eta \cos 2 \psi\right)=R^{2}\left(1+\eta^{2}+2 \eta \cos 2 \phi\right)$ (from Fig.3), $\cos 2 \phi=1-2 \sin ^{2} \phi$ and introduced notations [20]

$$
n^{2}=\frac{4 \eta}{(1+\eta)^{2}}<1, \quad k^{2}=\frac{4 \eta}{(1+\eta)^{2}+z^{2} / R^{2}}<1,
$$


which allow to write

$$
\begin{aligned}
& 1+\eta^{2}+2 \eta \cos 2 \phi=(1+\eta)^{2}\left(1-n^{2} \sin ^{2} \phi\right), \\
& r_{1}^{2}+z^{2}=R^{2}\left[(1+\eta)^{2}+\frac{z^{2}}{R^{2}}\right]\left(1-k^{2} \sin ^{2} \phi\right) .
\end{aligned}
$$

To express (21) in terms of complete elliptic integrals, let's decompose

$$
\frac{\left(1+\eta-2 \eta \sin ^{2} \phi\right)\left(1-k^{2} \sin ^{2} \phi\right)}{1-n^{2} \sin ^{2} \phi}=A+B\left(1-k^{2} \sin ^{2} \phi\right)+\frac{C}{1-n^{2} \sin ^{2} \phi} .
$$

For unknown coefficients $A, B, C$ we get a system of equations

$$
\begin{aligned}
& B n^{2}=2 \eta, \\
& A+B+C=1+\eta, \\
& A n^{2}+B\left(n^{2}+k^{2}\right)=2 \eta+(1+\eta) k^{2} .
\end{aligned}
$$

The solution of this system is easily found to be

$$
A=\frac{k^{2}}{2 n^{2}}\left(1-\eta^{2}\right), \quad B=\frac{2 \eta}{n^{2}}=\frac{(1+\eta)^{2}}{2}, \quad C=\frac{1}{2}\left(1-\eta^{2}\right)\left[1-\frac{k^{2}}{n^{2}}\right] .
$$

Given (21), (22), (24) and (26), the electrostatic potential (18) can be expressed in terms of complete elliptic integrals as follows (in full agreement with the results of [20]):

$$
\begin{aligned}
& \phi(\eta R, z)=\frac{\sigma R}{2 \pi \epsilon_{0}}\left[\frac{1-\eta^{2}}{\sqrt{(1+\eta)^{2}+z^{2} / R^{2}}} K(k)+\sqrt{(1+\eta)^{2}+z^{2} / R^{2}} E(k)+\right. \\
& \left.\frac{1-\eta}{1+\eta} \frac{z^{2} / R^{2}}{\sqrt{(1+\eta)^{2}+z^{2} / R^{2}}} \Pi\left(n^{2}, k\right)-\pi \frac{|z|}{R}\right] .
\end{aligned}
$$

Here

$$
\Pi\left(n^{2}, k\right)=\int_{0}^{\pi / 2} \frac{d \theta}{\left(1-n^{2} \sin ^{2} \phi\right) \sqrt{1-k^{2} \sin ^{2} \theta}}
$$

is the complete elliptic integral of the third kind [25, 26].

If the observation point $P$ is situated on the $z$-axis, then $\eta=0, k^{2}=n^{2}=0$, and using $K(0)=E(0)=\Pi(0,0)=\pi / 2$, (27) simplifies to the well-known expression

$$
\phi(0, z)=\frac{\sigma}{2 \pi \epsilon_{0}}\left(\sqrt{z^{2}+R^{2}}-|z|\right) .
$$

\section{Electric field above or below the disk}

Due to rather complicated character of the expression (27) for the electrostatic potential, it will be easier to calculate the components of the corresponding electric field using (18) instead of the integrated form (27). Namely, for the radial component of the electric field it follows from (18) that

$$
E_{r}(\eta R, z)=-\frac{1}{R} \frac{\partial}{\partial \eta} \phi(\eta R, z)=-\frac{\sigma}{2 \pi \epsilon_{0} R} \int_{0}^{\pi} \frac{r_{1}\left(\frac{\partial r_{1}}{\partial \eta}\right)_{\theta}}{\sqrt{r_{1}^{2}+z^{2}}} d \theta .
$$


In this expression, the partial derivative $\left(\frac{\partial r_{1}}{\partial \eta}\right)_{\theta}$ should be calculated with constant $\theta$. On the other hand, it is clear from the Fig.3 that

$$
r_{1}^{2}=R^{2}\left(1+\eta^{2}-2 \eta \cos 2 \psi\right),
$$

where $\psi$, as equation (19) indicates, is a function of both $\eta$ and $\theta$. Therefore,

$$
r_{1}\left(\frac{\partial r_{1}}{\partial \eta}\right)_{\theta}=r_{1}\left[\left(\frac{\partial r_{1}}{\partial \eta}\right)_{\psi}+\left(\frac{\partial r_{1}}{\partial \psi}\right)_{\eta}\left(\frac{\partial \psi}{\partial \eta}\right)_{\theta}\right]
$$

Differentiating (31), we get for partial derivatives

$$
r_{1}\left(\frac{\partial r_{1}}{\partial \eta}\right)_{\psi}=R^{2}(\eta-\cos 2 \psi), r_{1}\left(\frac{\partial r_{1}}{\partial \psi}\right)_{\eta}=2 \eta R^{2} \sin 2 \psi
$$

While it follows from (19) that

$$
\tan \theta\left[-2 \sin 2 \psi\left(\frac{\partial \psi}{\partial \eta}\right)_{\theta}-1\right]=2 \cos 2 \psi\left(\frac{\partial \psi}{\partial \eta}\right)_{\theta},
$$

and, therefore,

$$
\left(\frac{\partial \psi}{\partial \eta}\right)_{\theta}=-\frac{\tan \theta}{2(\cos 2 \psi+\tan \theta \sin 2 \psi)}=-\frac{\sin 2 \psi}{2(1-\eta \cos 2 \psi)}
$$

Substituting (33) and (35) into (32), we get

$$
r_{1}\left(\frac{\partial r_{1}}{\partial \eta}\right)_{\theta}=-\frac{R^{2} \cos 2 \psi\left(1+\eta^{2}-2 \eta \cos 2 \psi\right)}{1-\eta \cos 2 \psi} .
$$

Combined with (20) and (30), this result implies

$E_{r}=\frac{\sigma R}{\pi \epsilon_{0}} \int_{0}^{\pi / 2} \frac{\cos 2 \psi}{\sqrt{r_{1}^{2}+z^{2}}} d \psi=-\frac{\sigma}{\pi \epsilon_{0} \sqrt{(1+\eta)^{2}+z^{2} / R^{2}}} \int_{0}^{\pi / 2} \frac{1-2 \sin ^{2} \phi}{\sqrt{1-k^{2} \sin ^{2} \phi}} d \phi$.

But $\sin ^{2} \phi=\left[1-\left(1-k^{2} \sin ^{2} \phi\right)\right] / k^{2}$ and, respectively, (37) can be immediately expressed in terms of complete elliptic integrals of the first and second kinds (in agreement with [21]):

$$
E_{r}=-\frac{\sigma}{\pi \epsilon_{0} \sqrt{(1+\eta)^{2}+z^{2} / R^{2}}}\left[\left(1-\frac{2}{k^{2}}\right) K(k)+\frac{2}{k^{2}} E(k)\right] .
$$

The combination of complete elliptic integrals that appear in (38) can be expressed through the Legendre function of the second kind and half-integral order $Q_{1 / 2}$ (the so called toroidal function of zeroth order — see [27] and references therein). Indeed, we have [22, 28]

$$
Q_{1 / 2}\left(\frac{2}{k^{2}}-1\right)=\frac{2-k^{2}}{k} K(k)-\frac{2}{k} E(k) .
$$

Therefore (38) can be rewritten as follows:

$$
E_{r}=\frac{\sigma}{2 \pi \epsilon_{0} \sqrt{\eta}} Q_{1 / 2}\left(\frac{1+\eta^{2}+z^{2} / R^{2}}{2 \eta}\right) .
$$


It is not immediately clear that (9) is the $z \rightarrow 0$ limit of (38). However this follows from the identities

$$
K(n)=(1+\eta) K(\eta), \quad E(n)=\frac{2}{1+\eta} E(\eta)-(1-\eta) K(\eta)
$$

that by themselves can be obtained from Gauss' Transformation formulas for complete elliptic integrals (transformations 164.02 in [25]).

Similarly, for the vertical component of the electric field, we obtain after some simple manipulations

$$
\begin{array}{r}
E_{z}=-\frac{\partial \phi(\eta R, z)}{\partial z}=-\frac{\sigma}{2 \pi \epsilon_{0}}\left[\int_{0}^{\pi} \frac{z}{\sqrt{r_{1}^{2}+z^{2}}} d \theta-\pi \operatorname{sign}(z)\right]= \\
\frac{\sigma}{2 \pi \epsilon_{0}}\left[\frac{-z / R}{\sqrt{(1+\eta)^{2}+z^{2} / R^{2}}} \int_{0}^{\pi / 2} \frac{\frac{2}{1+\eta}-n^{2} \sin ^{2} \phi}{\sqrt{1-k^{2} \sin ^{2} \phi}\left(1-n^{2} \sin ^{2} \phi\right)} d \phi+\pi \operatorname{sign}(z)\right] .
\end{array}
$$

Here

$$
\operatorname{sign}(z)=\frac{d|z|}{d z}=\left\{\begin{aligned}
1 & \text { if } z>0 \\
0 & \text { if } z=0 \\
-1 & \text { if } z<0
\end{aligned}\right.
$$

Using $\frac{2}{1+\eta}-n^{2} \sin ^{2} \phi=\frac{1-\eta}{1+\eta}+1-\sin ^{2} \phi$, the integral in (42) can be immediately expressed in terms of the complete elliptic integrals, and the final answer is

$$
E_{z}=-\frac{\sigma}{2 \pi \epsilon_{0}}\left[\frac{z / R}{\sqrt{(1+\eta)^{2}+z^{2} / R^{2}}}\left(K(k)+\frac{1-\eta}{1+\eta} \Pi\left(n^{2}, k\right)\right)-\pi \operatorname{sign}(z)\right] .
$$

This answer does not look identical to the expression given in [21. However it coincides with the result obtained in [29] and it was shown in [29] that the results of [21] and [29] for $E_{z}$ are actually equivalent.

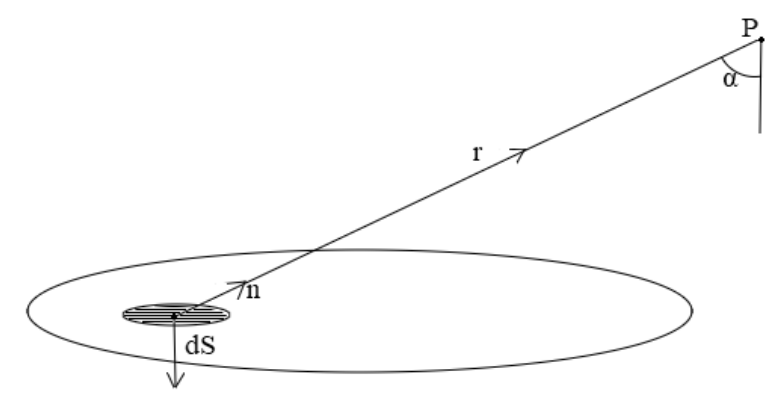

Figure 4. Definition of variables in the solid angle formula (45). $\vec{n}=\frac{\vec{r}}{r}$ is the unit vector in the direction of $\vec{r}$.

It follows from the solid angle formula (see Fig,4)

$$
\Omega=-\int \frac{\vec{n} \cdot d \vec{S}}{r^{2}}=\int \frac{\cos \alpha}{r^{2}} d S
$$

that the $z$-component of the gravitational attraction at $P$ of any plane homogeneous lamina with constant surface density is equal to the negative of surface density times 
the Newton's constant times the solid angle which the lamina subtends at $P$ [30]. In the case of our charged disk, this translates into

$$
E_{z}=\frac{\sigma}{4 \pi \epsilon_{0}} \Omega
$$

where $\Omega$ is the solid angle which the disk subtends at $P$. There exist independent calculations of $\Omega$ [31, 32. It can be verified that (for $z>0$ ) the solid angle $\Omega$ that follows from (44) and (46) is consistent with the results of [31, 32].

\section{Electrostatic potential and electric field at points outside the disk boundary}

If the projection point $P$ lies outside the disk boundary $(\eta>1)$, when we can arrange only one wedge crossing the disk, as shown in Fig.5. When $\theta$ changes from 0 to $\theta_{\max }$,

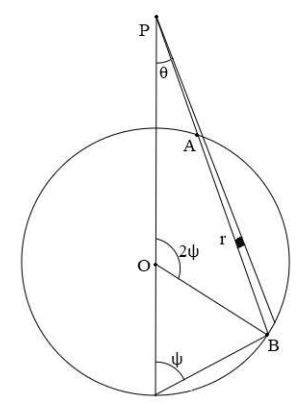

Figure 5. Definition of variables when the projection point $P$ lies outside the disk boundary.

the wedge covers half the disk. The limit angle $\theta_{\text {max }}$ corresponds the wedge touching the disk. Therefore $\sin \theta_{\max }=1 / \eta$. The other symmetric half of the disk makes an equal contribution to the electrostatic potential, and we can write

$\phi(\eta R, z)=\frac{\sigma}{2 \pi \epsilon_{0}} \int_{0}^{\theta_{\max }} d \theta \int_{r_{1}}^{r_{2}} \frac{r}{\sqrt{r^{2}+z^{2}}} d r=\frac{\sigma}{2 \pi \epsilon_{0}} \int_{0}^{\theta_{\max }}\left(\sqrt{r_{2}^{2}+z^{2}}-\sqrt{r_{1}^{2}+z^{2}}\right) d \theta$,

where $r_{1}=P A$ and $r_{2}=P B$ (see Fig 5 ). Both $r_{1}$ and $r_{2}$ obey the same quadratic equation $R^{2}=\eta^{2} R^{2}+r^{2}-2 \eta R r \cos \theta$ with solutions

$$
r_{1}=R\left[\eta \cos \theta-\sqrt{1-\eta^{2} \sin ^{2} \theta}\right], r_{2}=R\left[\eta \cos \theta+\sqrt{1-\eta^{2} \sin ^{2} \theta}\right] .
$$

In the first integral let's again introduce the angle $\psi$ as defined in Fig.5. Then $r_{2}^{2}=R^{2}\left(1+\eta^{2}-2 \eta \cos 2 \psi\right)$ and

$$
\sqrt{r_{2}^{2}+z^{2}}=R \sqrt{(1+\eta)^{2}+z^{2} / R^{2}} \sqrt{1-k^{2} \sin ^{2} \phi}
$$

where $\phi=\pi / 2-\psi$ and $k$ was defined in (22). In addition, Fig.5 indicates that

$$
\tan \theta=\frac{R \sin (\pi-2 \psi)}{\eta R+R \cos (\pi-2 \psi)}=\frac{\sin 2 \psi}{\eta-\cos 2 \psi},
$$


and, correspondingly,

$$
d \theta=2 \frac{\eta \cos 2 \psi-1}{1+\eta^{2}-2 \eta \cos 2 \psi} d \psi=2 \frac{1+\eta \cos 2 \phi}{1+\eta^{2}+2 \eta \cos 2 \phi} d \phi .
$$

Therefore

$$
\int_{0}^{\theta_{\max }} \sqrt{r_{2}^{2}+z^{2}} d \theta=\frac{2 R \sqrt{(1+\eta)^{2}+\frac{z^{2}}{R^{2}}}}{(1+\eta)^{2}} \int_{0}^{\frac{\pi}{2}-\psi_{m}} \frac{\left(1+\eta-2 \eta \sin ^{2} \phi\right)\left(1-k^{2} \sin ^{2} \phi\right)}{\left(1-n^{2} \sin ^{2} \phi\right) \sqrt{1-k^{2} \sin ^{2} \phi}} d \phi
$$

where $\psi_{m}$ corresponds to $\theta_{\max }$ and thus satisfies $\cos 2 \psi_{m}=\sin \theta_{\max }=1 / \eta$.

In the second integral of (47), we use an alternative definition of the angle $\psi$ shown in Fig.6. Then $r_{1}^{2}=R^{2}\left(1+\eta^{2}-2 \eta \cos 2 \psi\right)$, equations (50) and (51) are still valid, but

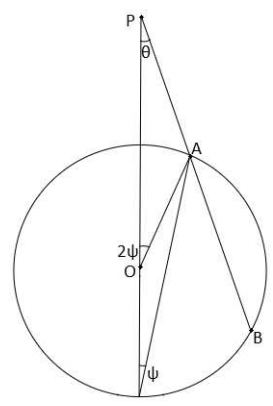

Figure 6. Alternative definition of the angle $\psi$.

now $\psi$ changes from 0 to $\psi_{m}$, when $\theta$ changes from 0 to $\theta_{\max }$, and, correspondingly, $\phi$ changes from $\pi / 2$ to $\pi / 2-\psi_{m}$. Therefore

$$
\int_{0}^{\theta_{\max }} \sqrt{r_{1}^{2}+z^{2}} d \theta=\frac{2 R \sqrt{(1+\eta)^{2}+\frac{z^{2}}{R^{2}}}}{(1+\eta)^{2}} \int_{\frac{\pi}{2}}^{\frac{\pi}{2}} \frac{\left(1+\eta-2 \eta \sin ^{2} \phi\right)\left(1-k^{2} \sin ^{2} \phi\right)}{\left(1-n^{2} \sin ^{2} \phi\right) \sqrt{1-k^{2} \sin ^{2} \phi}} d \phi .
$$

As we see,the difference of two integrals, (52) and (53), just gives the right-hand-side of (21), and, therefore the electrostatic potential when $\eta>1$ is still given by (27), but without the last, proportional to $|z|$ term. Introducing Heaviside step function $H(x)=(1+\operatorname{sign}(x)) / 2$, the final expression for the electrostatic potential, valid for all values of $\eta$, takes the form

$$
\begin{aligned}
& \phi(\eta R, z)=\frac{\sigma R}{2 \pi \epsilon_{0}}\left[\frac{1-\eta^{2}}{\sqrt{(1+\eta)^{2}+z^{2} / R^{2}}} K(k)+\sqrt{(1+\eta)^{2}+z^{2} / R^{2}} E(k)+\right. \\
& \left.\frac{1-\eta}{1+\eta} \frac{z^{2} / R^{2}}{\sqrt{(1+\eta)^{2}+z^{2} / R^{2}}} \Pi\left(n^{2}, k\right)-\pi H(1-\eta) \frac{|z|}{R}\right] .
\end{aligned}
$$

The radial electric field is still given by (40), except that the derivative of Heaviside step function will bring an additional term proportional to the Dirac delta function:

$$
E_{r}=\frac{\sigma}{2 \pi \epsilon_{0} \sqrt{\eta}} Q_{1 / 2}\left(\frac{1+\eta^{2}+z^{2} / R^{2}}{2 \eta}\right)-\frac{\sigma}{2 \epsilon_{0}} \delta(1-\eta) \frac{|z|}{R} .
$$


Finally, the vertical component of the electric field, valid for all values of $\eta$, has the form

$$
E_{z}=-\frac{\sigma}{2 \pi \epsilon_{0}}\left[\frac{z / R}{\sqrt{(1+\eta)^{2}+z^{2} / R^{2}}}\left(K(k)+\frac{1-\eta}{1+\eta} \Pi\left(n^{2}, k\right)\right)-\pi H(1-\eta) \operatorname{sign}(z)\right] .
$$

To use these formulas near the rim of the disk $(\eta \approx 1)$, the following asymptotic expansions [25], valid when $n^{2} \approx 1$, are useful:

$$
K(n) \approx \ln 4-\ln \left(1-n^{2}\right), \quad \Pi\left(n^{2}, k\right) \approx K(k)-\frac{E(k)}{1-k^{2}}+\frac{\pi\left(2-k^{2}-n^{2} k^{2}\right)}{4 \sqrt{\left(1-n^{2}\right)\left(1-k^{2}\right)^{3}}} .
$$

Then it can be seen from (9) that the radial electric field has a logarithmic singularity at the rim of the disc. This singularity is related to the discontinuity in the surface charge density at $\eta=1$ and can be avoided by using a distribution of charge with surface density that falls to zero at the rim [22].

\section{Concluding remarks}

Disc-shaped structures are widespread in astrophysics, their formation being associated with the conservation of angular momentum in gas flow around compact gravitating objects [33]. It is not surprising therefore that the gravitational counterpart of the considered problem and its generalization to the case of non-uniform disks attracted much more attention (see, for example, [18, 19, 20, 21, 22, 29, 34, 35, 36, 37, 38] and references cited therein).

In pedagogical literature, one can find considerations of the gravitational field of a massive ring [39, 40, 41], and of the electric field of a homogeneous ring [27, 42, 43, 44, 45]. However, we were able to find only one paper [46] discussing the gravitational field of a homogeneous massive disk.

In [46] the gravitational field of a hypothetical flat Earth was considered in the approximation $\eta \ll 1 \pm$. To get the radial electric field in the $\eta \ll 1$ approximation from (40), we use the expansion [27, 50]

$Q_{1 / 2}(\beta)=\frac{\pi}{2(2 \beta)^{3 / 2}} \sum_{n=0}^{\infty} \frac{(4 n+1) ! !}{2^{2 n}(n+1) ! n !} \frac{1}{(2 \beta)^{2 n}}=\frac{\pi}{2(2 \beta)^{3 / 2}}\left(1+\frac{15}{8} \frac{1}{(2 \beta)^{2}} \ldots\right)$,

with $2 \beta=\left(1+\eta^{2}+z^{2} / R^{2}\right) / \eta$. As a result we get

$$
E_{r} \approx \frac{\sigma \eta}{4 \epsilon_{0}\left(1+z^{2} / R^{2}\right)^{3 / 2}}\left[1+\frac{3\left(1-4 z^{2} / R^{2}\right)}{8\left(1+z^{2} / R^{2}\right)^{2}} \eta^{2}\right] .
$$

$\ddagger$ You will not believe, there are many people nowadays who think that the Earth is flat. They even hold their own Flat Earth International Conferences [47. Alas, calculations, as in [46, cannot change their mind - they do not believe in gravity. For every educated person, starting from the ancient times of Greek and Roman writers, the sphericity of the Earth was an indisputable fact based on solid empirical data [48, 49. The fact that flat Earth proponents frantically and ignorantly attack this ancient knowledge is an alarming sign of the growing irrationality and decline of faith in the scientific method in modern society [47]. 
For the vertical component of the electric field, we use expansions [25]

$$
\begin{gathered}
K(k)=\frac{\pi}{2}\left[1+\frac{1}{4} k^{2}+\frac{9}{64} k^{4}+\ldots\right], \Pi\left(n^{2}, k\right)=\frac{\pi}{2} \sum_{m=0}^{\infty} \sum_{j=0}^{m} \frac{(2 m) !(2 j) ! k^{2 j} n^{2(m-j)}}{4^{m} 4^{j}(m !)^{2}(j !)^{2}}= \\
\frac{\pi}{2}\left[1+\frac{1}{2} n^{2}+\frac{1}{4} k^{2}+\frac{3}{8} n^{4}+\frac{1}{16} k^{2} n^{2}+\frac{9}{64} k^{4}+\ldots\right],
\end{gathered}
$$

and get in the $\eta \ll 1$ approximation (for $z>0$ ):

$$
E_{z} \approx \frac{\sigma}{4 \epsilon_{0}}\left[2\left(1-\frac{z / R}{\sqrt{1+z^{2} / R^{2}}}\right)-\frac{3 z / R}{2\left(1+z^{2} / R^{2}\right)^{5 / 2}} \eta^{2}\right] .
$$

Under substitution $1 /\left(4 \pi \epsilon_{0}\right) \rightarrow-G, G$ being the Newton gravitational constant, (59) and (61) reproduce the results of [46].

In fact, the problem addressed in this note was resolved a long time ago, much earlier than is commonly thought. Conway notes in [51] that the fact that the potential of a homogeneous disk is expressible in terms of elliptic integrals was already known to Weber in 1873 [52]. However, Weber does not provide any reference.

The footnote in [19] gives a hint that according to Todhunter [53] Giovanni Antonio Amedeo Plana calculated gravitational pulls from the ring and from the circular disk. We were able to find Plana's publication [54]. In it, Plana indeed gives the gravitational attraction of a homogeneous circular disk in terms of complete elliptic integrals. The corresponding expression for the gravitational potential was given much later by Arthur Cayley [55].

It seems that the contributions of Plana and Cayley have now been forgotten, because all the modern articles that we checked cite [17, 20, 21] as primary sources where the problem of a homogeneous disk was solved. We think that the upcoming bicentennial anniversary of the Giovanni Plana's paper [54] is a good occasion both to restore the legacy of Plana and Cayley, and to make this venerable problem accessible to a wide audience of physics students.

\section{References}

[1] Griffiths D J 2013 Introduction to electrodynamics (New York: Pearson Education)

[2] Franklin J 2005 Classical electromagnetism (New York: Pearson Education)

[3] Ohanian H C 1988 Classical electrodynamics (Boston: Allyn and Bacon)

[4] Vanderlinde J 2004 Classical electromagnetic theory (Dordrecht: Kluwer Academic Publishers)

[5] Reitz J R, Milford F J 1960 Foundations of electromagnetic theory (Reading: Addison-Wesley)

[6] Purcell E M, Morin D J 2013 Electricity and magnetism (Cambridge: Cambridge University Press)

[7] Jackson J D 1999 Classical electrodynamics (New York: John Wiley \& Sons)

[8] Smythe W R 1989 Static and dynamic electricity (London: Taylor and Francis)

[9] Greiner W 1998 Classical electrodynamics (New York: Springer-Verlag)

[10] Zangwill A 2013 Modern electrodynamics (Cambridge: Cambridge University Press)

[11] Schwartz M 1972 Principles of electrodynamics (New York: McGraw-Hill)

[12] Konopinski E J 1981 Electromagnetic fields and relativistic particles (New York: McGraw-Hill)

[13] Good R H, Nelson T J 1974 Classical theory of electric and magnetic fields (New York: Academic Press) 
[14] Panofsky W K H, Phillips M 1962 Classical electricity and magnetism (Reading: Addison-Wesley)

[15] Stratton J A 2007 Electromagnetic theory (Hoboken: John Wiley \& Sons)

[16] Eyges L 2012 The classical electromagnetic field (New York: Dover)

[17] Durand E 1953 Électrostatique. Vol. I. Les distributions (Paris: Masson et Cie)

[18] Duboshin G N 1961 The theory of attraction (Moscow: Fizmatlit) (in Russian)

[19] Kondrat'ev B P 2007 Potential theory: new methods and problems with solutions (Moscow: Mir) (in Russian)

[20] Lass H, Blitzer L 1983 The gravitational potential due to uniform disks and rings Celestial Mech. $30225-228$

[21] Krogh F T, Ng E W, Snyder W V 1982 The gravitational field of a disk Celestial Mech. 26 395-405

[22] Conway J T 2002 Analytical solutions for the Newtonian gravitational field induced by matter within axisymmetric boundaries Mon. Not. Roy. Astron. Soc. 316 540-554

[23] Routh E J 1922 A treatise on analytical statistics, volume 2 (Cambridge: Cambridge University Press)

[24] Friedberg R 1993 The electrostatics and magnetostatics of a conducting disk Am. J. Phys. 61 1084-1096

[25] Byrd P F, Friedman M D 1971 Handbook of Elliptic Integrals for Engineers and Scientists, Second Edition (Berlin: Springer-Verlag)

[26] Abramowitz M, Stegun I A 1972 Handbook of Mathematical Functions (New York: Dover)

[27] Selvaggi J, Salon S, Chari M V K 2007 An application of toroidal functions in electrostatics Am. J. Phys. 75 724-727

[28] Snow C 1954 Formulas for Computing Capacitance and Inductance (Washington: National Bureau of Standards circular 544)

[29] Fukushima T 2010 Precise computation of acceleration due to uniform ring or disk Celest. Mech. Dyn. Astron. 108 339-356

[30] Kellogg O D 1967 Foundations of Potential Theory, Berlin (Berlin: Springer-Verlag)

[31] Paxton F 1959 Solid Angle Calculation for a Circular Disk Rev. Sci. Instrum. 30 254-258

[32] Conway J T 2010 Analytical solution for the solid angle subtended at any point by an ellipse via a point source radiation vector potential Nucl. Instrum. Meth. A 613 17-27

[33] Regev O, Umurhan O M, Yecko P A 2016 Modern Fluid Dynamics for Physics and Astrophysics (New York: Springer).

[34] Eckhardt D H, Pestaña J L G 2002 Technique for Modeling the Gravitational Field of a Galactic Disk $A p J \mathbf{5 7 2}$ L135-L137

[35] Fukushima T 2016 Numerical computation of gravitational field of infinitely thin axisymmetric disc with arbitrary surface mass density profile and its application to preliminary study of rotation curve of M33 Mon. Not. Roy. Astron. Soc. 456 3702-3714

[36] Huré J M 2012 A key-formula to compute the gravitational potential of inhomogeneous discs in cylindrical coordinates Celest. Mech. Dyn. Astr. 114 365-385

[37] Nieto M M 2005 Analytic Gravitational-Force Calculations for Models of the Kuiper Belt, with Application to the Pioneer Anomaly Phys. Rev. D 72, 083004

[38] Pierens A, Huré J M 2004 Rotation Curves of Galactic Disks for Arbitrary Surface Density Profiles: A Simple and Efficient Recipe ApJ 605 179-182

[39] Schumayer D, Hutchinson D A W 2019 Peculiarities in the gravitational field of a filamentary ring Am. J. Phys. 87 384-394

[40] Tobin R W, West J 2006 Close orbits about a massive thin ring Eur. J. Phys. 27 215-223

[41] West J, Dassanayake S, Daniel A 1998 Bound orbits with positive energy Am. J. Phys. 66, 25-28

[42] Zypman F R 2006 Off-axis electric field of a charged ring Am. J. Phys. 74 295-300

[43] Noh H R 2017 Electrostatic potential of a charged ring: Applications to elliptic integral identities J. Korean Phys. Soc. $\mathbf{7 1} 37-41$

[44] Datta S 2007 Electric and magnetic fields from a circular coil using elliptic integrals Phys. Educ. India 24 203-212 
[45] Ciftja O, Babineaux A and Hafeez N 2009 The electrostatic potential of a uniformly charged ring Eur. J. Phys. 30 623-627

[46] Kuzii O, Rovenchak A 2019 What the gravitation of a flat Earth would look like and why thus the Earth is not actually flat Eur. J. Phys. 40035008

[47] McIntyre L 2019 Calling all physicists Am. J. Phys. 87 694-695

[48] Dreyer J L E 1953 A History of Astronomy from Thales to Kepler (New York: Dover Publications)

[49] Russell J B 1991 Inventing the Flat Earth: Columbus and Modern Historians (New York: Praeger)

[50] Selvaggi J, Salon S, Kwon O, Chari M V K 2004 Calculating the external magnetic field from permanent magnets in permanent-magnet motors - An alternative method IEEE Trans. Magnetics 40 3278-3285

[51] Conway J T 2016 Vector potentials for the gravitational interaction of extended bodies and laminas with analytical solutions for two disks Celest. Mech. Dyn. Astr. 125 161-194

[52] Weber H 1873 Ueber die Besselschen functionen und ihre anwendung auf die theorie der elektrischen ströme J. Reine Angew. Math. 75 75-105

[53] Todhunter I 1873 History of the Mathematical Theories of Attraction and the Figure of the Earth (London: Constable \& Company)

[54] Plana J 1820 Solution de différents problémes relatifs á la loi de la résultante de l'attraction exercée sur un point matériel par le cercle, les couches cylindriques, et quelques autres corps qui en dépendent par la forme de leurs éléments Mémorie della Reale Accademia delle Scienze in Torino 24 389-450 https://www.biodiversitylibrary.org/item/32409\#page/461/

[55] Cayley A 1875 On the Potential of the Ellipse and the Circle Proc. Lond. Math. Soc. 6 38-58 